\title{
An Interactive UI Prototype Design for Enhancing On-site Museum and Art Gallery Experience through Digital Technology
}

\author{
Richard Yu-Chang Li and Alan Wee-Chung Liew \\ School of Information and Communication Technology, Griffith University, QLD, Australia
}

\begin{abstract}
Digital curation is an emerging topic of interest in museum studies. Onsite museum experience enhanced by digital technology can not only promote the social and educational roles of traditional museum in the digital era but also boost the value of cultural heritage more than ever before. Our objective is to design an on-site personalized museum recommendation system to enhance factors that extend the connection between visitors and collections, focusing on the aspect of user experience. The prototype is made up of two different functional components, a mobile user interface (UI) at the front-end for visitors to interact with the collections during their visit, and a collection database in the back-end where information is stored and accessed using semantic technology. In this article, we discuss the design of the UI interface for the collection of Australian paintings in the National Gallery of Australia, and evaluate the framework for visitor behaviour capture and analysis using the designed interface, thereby offering a system for establishing a personalized tour trajectory.
\end{abstract}

Keywords: Digital Museum; Digital Curation; User-oriented UI interface; Semantic Web; Resource Description Framework (RDF); Cognitive Walkthrough

\section{Introduction}

With the rapid increase in user population, mobile and wearable computing devices such as widescreen smartphones, Google glasses, and smart watches are increasingly being developed as common platforms for various applications. In this article, we describe the design of a mobile prototype for enhancing the factors of interaction, personalization, education, and visualization between visitors and collections in museums and art galleries, focusing on the aspect of visitor experience from an institutional viewpoint. The prototype consists of two modules, an iOS based mobile user interface (UI) with mobile Augmented Reality (AR) in 
association with Quick Response code (QR code) at the front-end, and a database which stores information about the collections using semantic technology at the back-end. In this article, we discuss the design of the user interface module targeted for Australian paintings in the National Gallery of Australia. The design framework is evaluated by Cognitive Walkthrough method to show how visitor's behaviour can be detected by enhanced interaction, thereby offering a recommendation system for a personalized visiting experience.

\section{Background}

Digital curation is an emerging topic of interest in museum studies. According to Tsapatori's 2003 research (as cited in Wojciechowski et al. 2004), the techniques of Virtual Reality (VR) and AR have been piloted to enhance the exhibitions in over a quarter of European museums. Many research projects and organizations are concentrating on building their digital platform using information technologies to compensate for the weakness of traditional on-site museums where an invisible gap exists between visitors and collections. Examples are the Ripoll Monastery exhibition at the National Art Museum of Catalonia (Andujar, Chica, and Brunet 2012), the virtual showcase (Bimber, Encarnação, and Schmalstieg 2003), and the Augmented Representation of Cultural Objects (ARCO) (Wojciechowski et al. 2004). Previously, the display platforms were often dominated by large complex equipment such as projectors or wide screen monitors. However, with the continuous improvement of mobile hardware and their penetration rate, some major museums have turned their digital display platforms from using fixed, immobile hardware to flexible portable devices. The Google Android and Apple iOS have become the common technology platforms for visitors to experience digital collections.

According to the latest report from IDC (http://www.idc.com/prodserv/smartphone-os-market-share.jsp), Android has the largest 
market share in mobile operating system, leading iOS by a large margin. However, Economou and Meintani (2011) observed that most museum apps are designed exclusively for iOS devices. Therefore, in order to ensure the widest compatibility in the museum and art gallery communities, our prototype is designed based on the iOS platform. Our prototype has several major components: a friendly UI interface with QR code reader, which provides extensive information about the collections to enrich visitor's experience; an Augmented Reality narrator, which plays an interactive role between visitors and collections; and a back-end semantic database, which store the collection's data and communicate with the front-end to make personalized recommendations during a visit.

Beer (1987) pointed out that museum visitors spend less than one minute on each collection on-site. In this work, our goal is to extend the time of artefact appreciation by enhancing the factors of interaction and personalization using digital mobile devices, thereby enhancing a visitor's visiting experience. If on-site museum experience can be enhanced by digital technologies, the development of digital curation in cultural expression will not only promote the social and educational roles of museums in this digital era but also boost the value of cultural heritage more than ever before. Clearly, leveraging the factors of UI design and semantic database can offer a valuable approach to assist curators and developers in structuring a useful digitization framework. Hence, in order to underline our statements, this article is organized as follows. We first point out the history of museum electronic devices and discuss several previous works on museum digitalization. We then describe our system architecture including the UI design and semantic database. An evaluation of the system using the Cognitive Walkthrough method will be presented. Finally, we conclude with some possible future development. 


\section{The history of museum handheld devices and previous work}

Audio guide and Multimedia guide are the two most common solutions in museums and art galleries in which visitors are able to concentrate on the appreciation of exhibits while receiving deeper information at the same time. In the early years, most of the approaches were based on bespoke systems which have full control of the system framework, layout design, as well as the content structure for each exhibit. According to Tallon and Walker (2008), Stedelijk audio guide, a closed-circuit short wave radio broadcasting system, was the first museum handheld device. It was brought in by a Dutch typographer and museum curator Willem Sandberg in 1952 for a high-profile short-term exhibition named "Vermeer: Real or Fake"' at Stedelijk Museum. The introduction of this approach was to provide foreign language tour to visitors during the exhibition. The device was initially introduced by Philips, a Dutch electronics company, for cinemas as a service to the hard-of-hearing. Tallon described the entire system as consisted of several functional components: a series of on-site loop-aerials, which were pre-installed into the skirting board around the outside of the galleries; a broadcast station, which was served by an analogue playback tape recorder; and a portable radio receiver, which was equipped with headphones for visitors. When visitors enter the audio-capture loop zone inside the museum, the broadcast station will transmit the audio through loop aerials to the receiver. In this early approach, all visitors will receive the same foreign language recording, which was broadcasted concurrently. Therefore, a PA (Public Address system) was required to broadcast the start of each record. In terms of the novel experience this service provided, it redefined the visitor's relationship with the museum.

Moving to the early nineties, with the constant improvement of technology, the museum device has changed from a simple audio provider to a multi-functional media presenter. The Tate Modern museum was one of the early examples, which provides a multimedia tour called The Tate Modern Multimedia Tour (MMT) that offers a wide range of 
digital contents by means of audio, video, and interactive application via PDA. It was sponsored by Bloomberg, in collaboration with Antenna Audio in 2002. The device provides a 45 minutes pre-set tour, and works with on-site wireless network for receiving the up-to-date information from a local server. It also detects a visitor's physical location so that he/she will no longer need to input the reference number of a collection to obtain additional information about the collection. The initial purpose of this attempt was to observe the effectiveness of wireless technology in the museum environment for improving the museum digitization (Proctor and Burton 2004).

Through the decades of development in portable devices for museum and art gallery, technological obsolescence has gradually militates against the adoption of bespoke system. The close system soon revealed its weakness when authorities need to focus their attention on both hardware and contents due to the limited budget. Many organizations and curators have therefore switched their focus to smartphone devices with customized apps. Some well-known techniques such as VR, AR, QR code, and html5 have soon been used widely to improve the capability of mobile applications (Ceipidor et al. 2013). However, according to the survey of Museums \& Mobile in 2013, most of the mobile-based museum apps are developed to be an information provider without back-end supports. The functions of pre-recorded audio tour remain the most common feature, which offers limited information related to the exhibits and collections by associating with building plans and pre-loading videos (Tallon 2013). Typical examples of these are the virtual museum of The Louvre on iPhone (LeVitus 2010), The State Hermitage Museum in Saint Petersburg, and the Love Lace exhibit at the Sydney Powerhouse Museum (Weiner 2011).

Despite the majority of museums and art galleries applications emphasizing the capability of information feeding, several recent projects did focus on the visitor-based interaction with some degree of personalization. For example, Solar Equation, a large-scale 
public art installation, was a part of the 2010 Light in Winter Festival in Federation Square, Melbourne. According to Lynch (2011), this art presentation is a result of a faithful simulation of the Sun, 100 million times smaller than the real thing. Through the pre-downloaded application on iOS devices, visitors are able to interact with the installation by disturbing the animations on the reflected surface in real-time and select different visualizations instead. By changing the surface animation, this attempt has implemented practical interaction between participants and collections.

Another example is StreetMuseum, an outdoors AR mobile app launched by the Museum of London (Hoare and Sorensen 2012). With this application, visitors can experience the city appearance by manipulating historical images in AR environment via their iPhone screen in over 200 sites of London City. The motivation was to bring the museum's photographic collections and artefacts alive by allowing people to look through their mobile screen and see how the past emerges. Similarly, the American Museum of Natural History has presented a mobile AR tool specifically designed for one of the exhibitions called Beyond Planet Earth: The Future of Space Exploration. The application allows visitors to detect 11 AR markers throughout the museum, and mark their favourite collections before share their opinions and experiences via social network (BRUSTEIN 2011). In this approach, limited personalization and interaction have been addressed.

\section{System architecture}

In the previous section, we have outlined several digitization efforts by discussing their implementations in cultural organizations. Unfortunately, most of these approaches focus little on the interactive aspect and with limited passive personalization such as favourite marking. Here we illustrate a new approach in which the personalization feature will be achieved proactively. The architecture is designed and developed under the User-Centered Design (UCD) 
principle, which asserted that 'the product should suit the user, rather than making the user suit the product' (Courage and Baxter 2005). In line with this statement, the purpose of our system is to collect visitor's interest quietly using intelligent technology when they interact with the front-end. As can be seen from Figure 1, a visitor's interest can be captured through the front-end interface. The results will then be sent to a semantic database as keywords via intermediate web service in which the entire visiting history will be recorded. When a new keyword is detected, the data retrieval process will be activated by the database, which stored the collections information using Resource Description Framework (RDF) format. Finally, the semantic reasoner infers the collections of interest from the input query and output a recommended collection with its location information back to the front-end. At this stage, a cycle has been completed and a new cycle will start, and a personalized tour path can therefore be established.

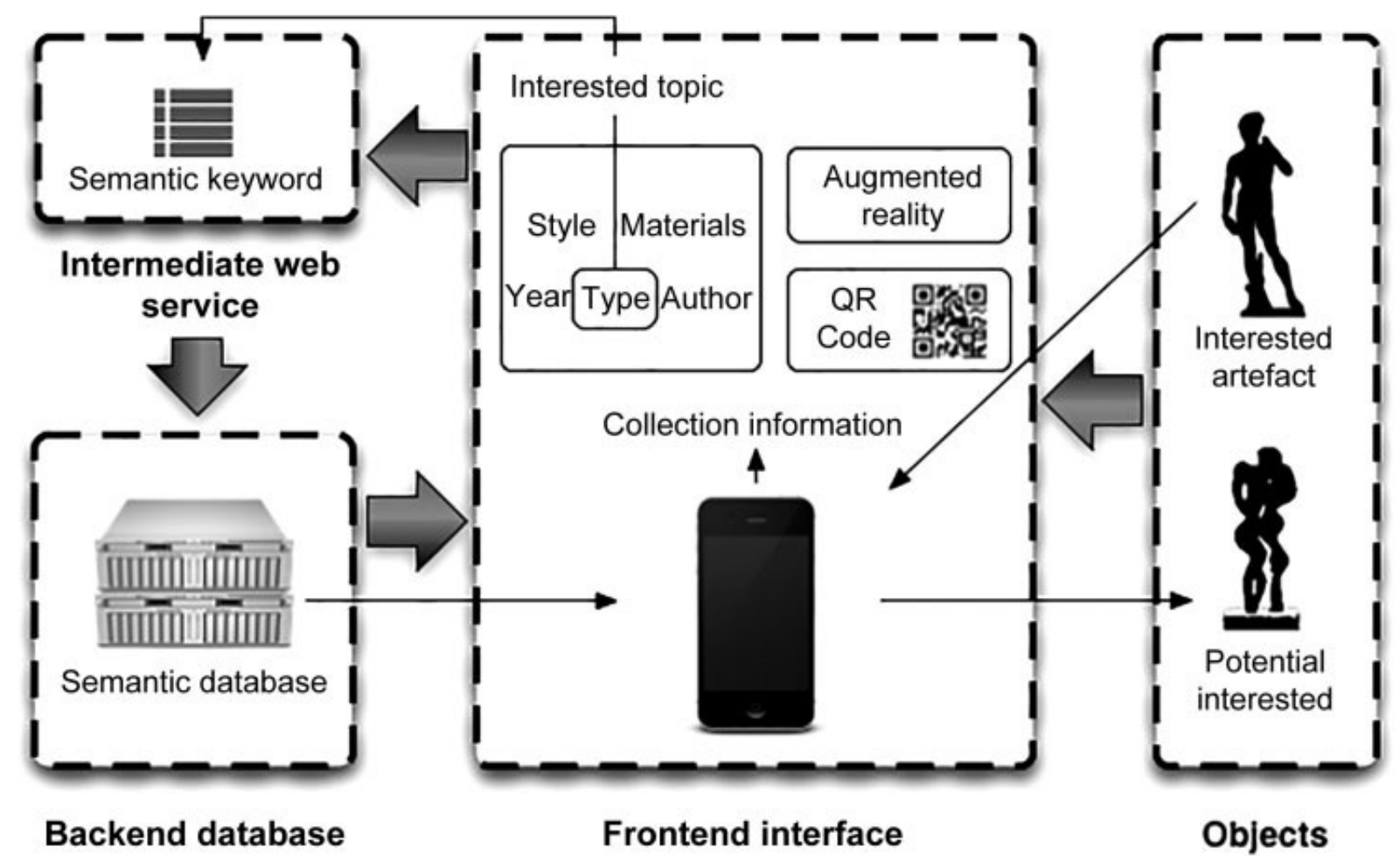

Figure 1. The system architecture. 
Figure 2 shows how data flows through the system. In order to structure this implementation systematically, the framework of the prototype is divided into two different modules: (i) a user friendly interface design at the front-end, which consists of the technologies of a mobile AR platform with a QR code reader; and (ii) a semantic database with a web based input interface at the back-end, where the ontologies of CIDOC Conceptual Reference Model (CIDOC-CRM) is adopted with Apache Jena-2 to store collections information.

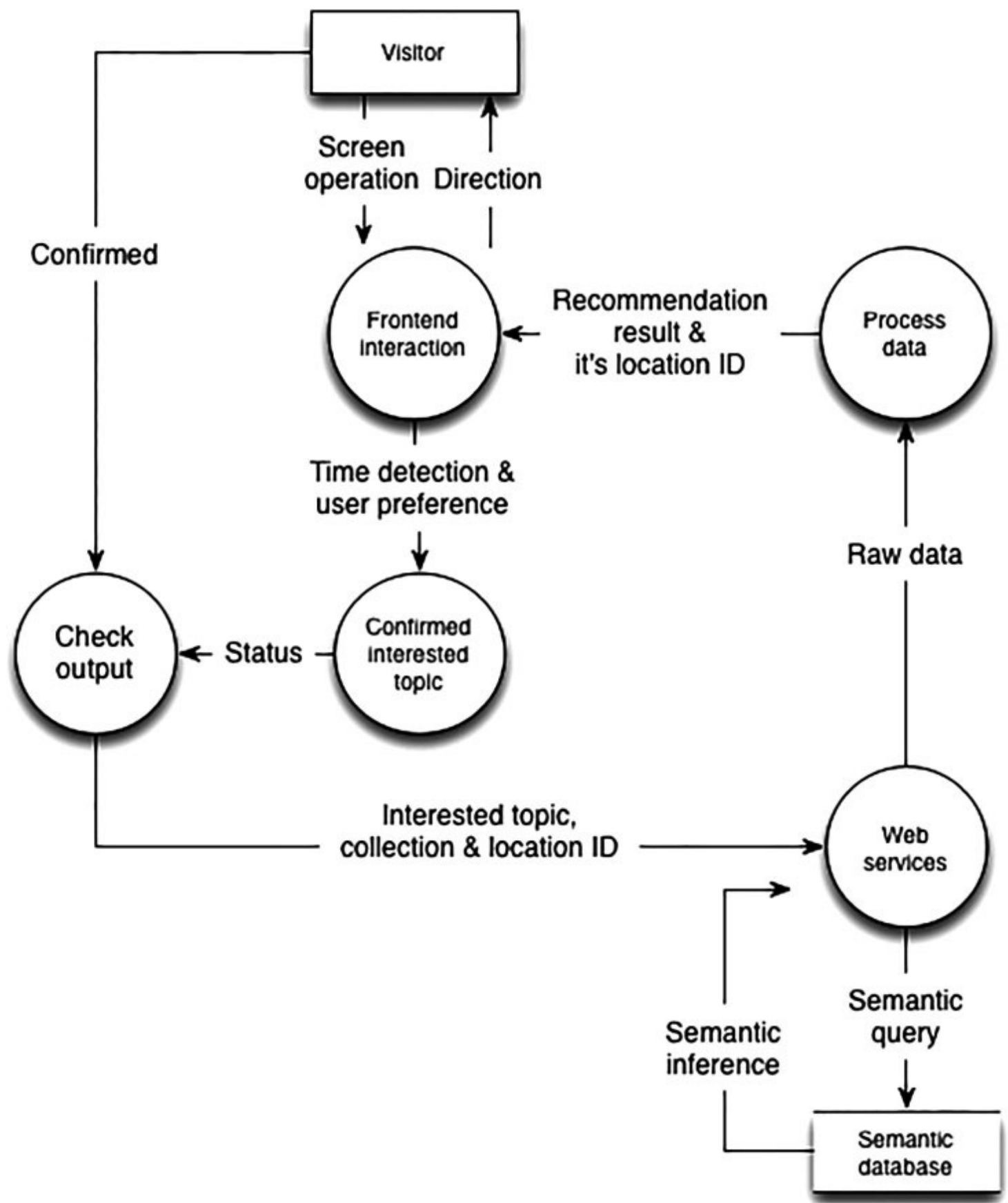

Figure 2. The data flow diagram. 


\section{UI design}

Oppermann (2002) stressed that a user friendly interface is a central issue for the usability of a software product. UI design can be regarded as one of the most important challenge in this project. In order to achieve ease of use and support intuitive manipulation, this system provides four main functionalities for interacting with the museum collections: a mobile platform-based interface with various theme pages displaying the detailed information related to the different topics for each collection; a step-by-step interactive quiz dialogs that detect the visitor's interest; a QR code-based navigation guide with 3D virtual interior viewpoints and floor maps displaying location information between collections; and a narrator in the mobile AR environment for enhancing visitor interaction.

Mobile-platform based User Interface - As shown in Figure 3, the interface is designed with high contrast display due to the on-site museum environment, where indirect lighting has often been used to protect the collections. With a high contrast display, visitors can read the information easily. The theme pages present the information in each topic for the collections. In this system, each collection is divided into five different topics: the name of the author; the year of creation; the type of materials used; the style and size of collection. According to Carretti (2007), imagery is one of the effective strategies to make items more salient. A storytelling theme page could assist visitors to understand the collections more quickly and significantly enriches their experience. For instance, the art movement of Modernism in Australia could be related to the theme that displays the period between the First and Second World War (Figure 3B). 
(a)

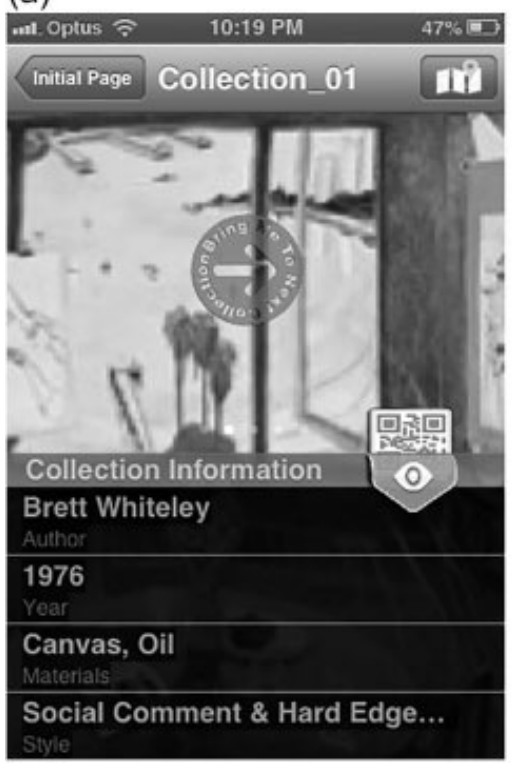

(b)

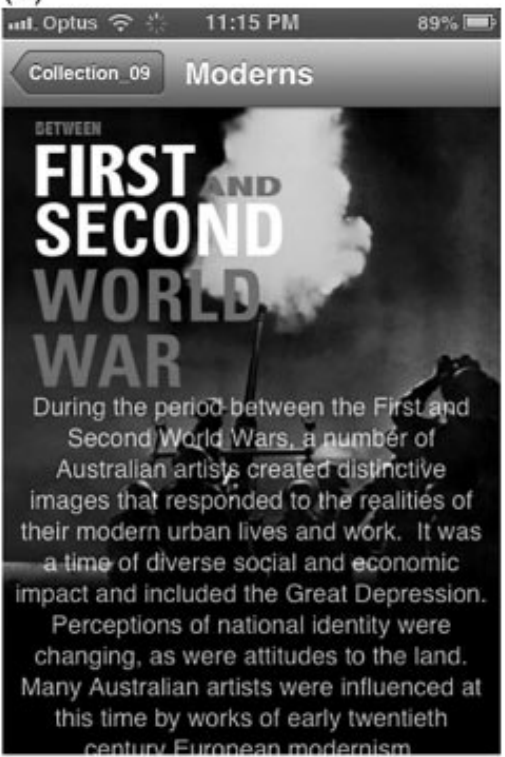

(c)

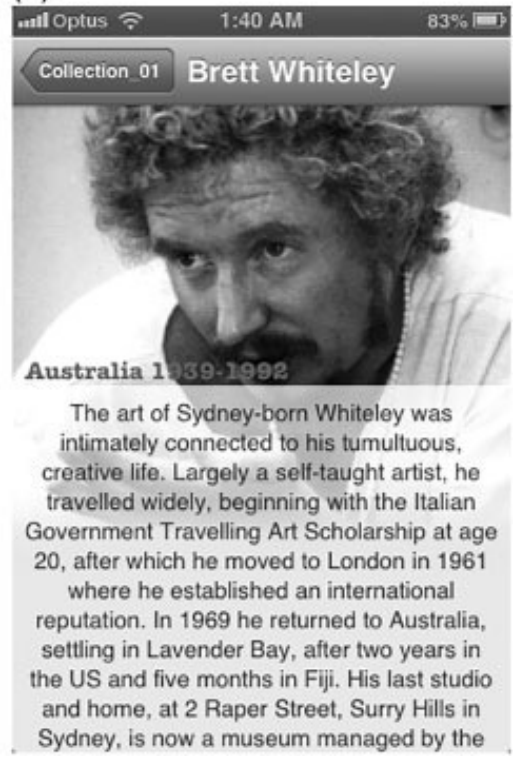

Figure 3. UI design.

Interactive quiz dialogs - With the intention of discovering visitor's interest, a series of interactive dialogs are used to interact with visitor during the visit. Figure 4 illustrates the process of recommendation when system detected "Year" as the collection preference. Tallon (2008) pointed out that screen-based devices may interrupt visitor experience from the exhibits, so only three dialogs are provided to minimize unwanted distraction. When a visitor triggers the button of "Bring Me To Next Collection" on the main screen of each collection, an observation dialog will appear as in Figure 4B if an inference about the visitor's interest can be made. , Otherwise, the dialog in Figure 4A will be shown instead. If the "YES" button is selected at the observation stage, the recommended collection with location information will be fed back to the visitor as shown in Figure 4C. Otherwise the "No" button will return the dialog to the detecting mode. When the 'GO' button is pressed, the visitor will then be guided by the 3D interactive map with location information as shown in Figure 5 to the next object. Through the iterative operation of the quiz dialogues, the entire personalized tour trajectory will eventually be established. 
(a)

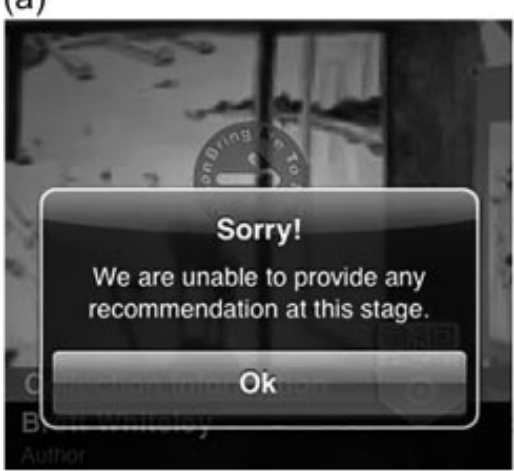

(b)

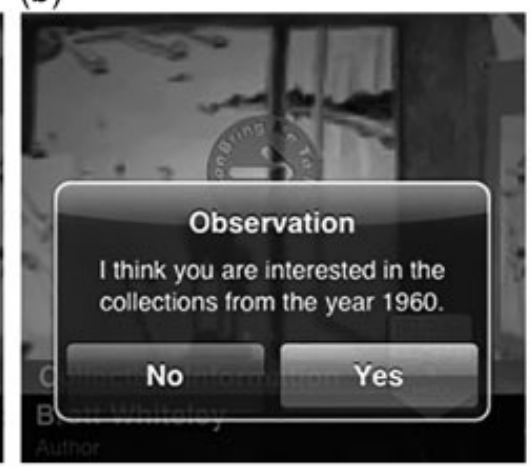

(c)

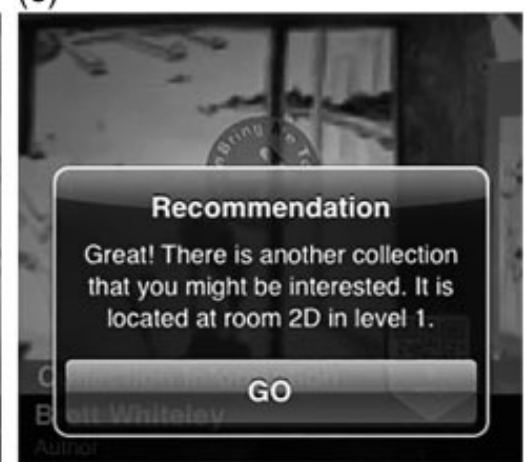

Figure 4. Interactive quiz dialogs.

Interactive virtual map navigation - Navigation has been recognized as a crucial task when interacting with virtual environments (Wang, Chiang, and Ho 2012). In this system, an HTML5 based interactive virtual museum viewpoint is introduced as shown in Figure 5 for visitors to target on-site locations by tagging QR codes placed around the museum. Through touch screen manipulation, visitors can access the location of interested collections without missing the track. As Falk (1992) commented, the time actually devoted to art appreciation is only around fifteen to forty minutes during a typical art museum visit, successfully reducing the time spent on searching for collections could improve the museum experience for the first-timer and occasional visitors. 


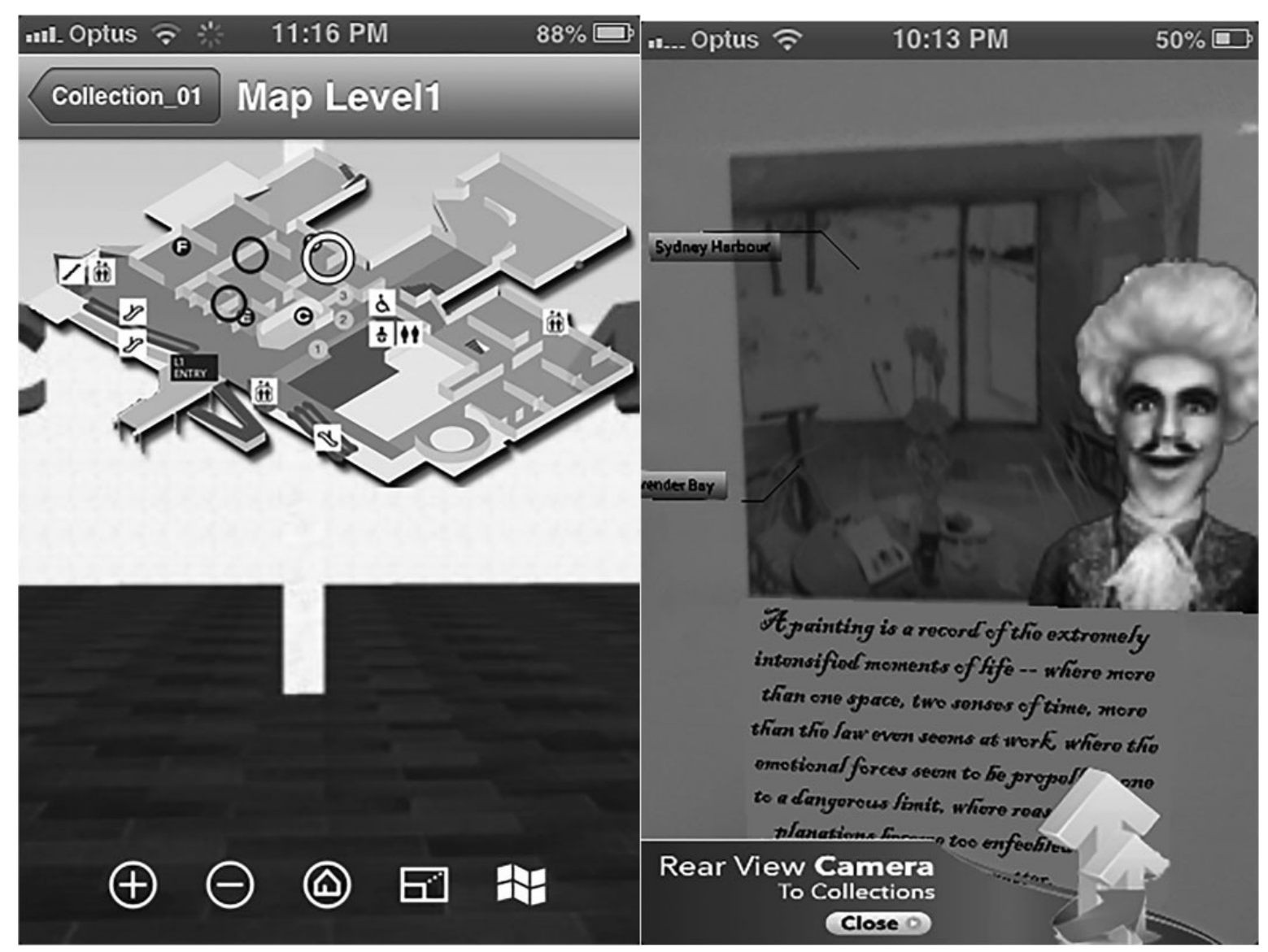

Figure 5. Interactive map navigation. Figure 6. Augment Reality presentation.

Mobile AR virtual agent - The use of AR on mobile platform has provided a highly interactive experience (Angelopoulou et al. 2011). As can be seen in Figure 6, an AR based narrator with markerless tracking technique will be adopted as one of the indicators that detect visitor's interest to interact with the on-site environment. In our system, each on-site collection serves as a marker during the visit. When detection is triggered via camera equipped hand-held devices, a 3D virtual agent with the collection's information will be displayed around the collection dynamically on the screen. The agent will act as a personal tour guide and play a storytelling audio pre-recorded by museum curators, exhibits organisers or artist themselves. This design not only provides another novel aspect for enhancing museum interaction but also avoids the need to key in a reference number. 


\section{Semantic database and intermediate web service}

The development of World Wide Web and the Internet unveil a new epoch for people to search and share information. However, with the huge amounts of information, many researchers advocate that the information needs to be personalized to boost its value (Huynh, Mazzocchi, and Karger 2007; Schreiber 2010). In line with this consideration, we apply semantic technology in co-operation with web services in our system to provide personalized information during a museum visit. This allows the system to achieve the goal advocated by Bowen and Filippini-Fantoni's (2004), that "the museum monologue" can be changed into "a user-centred information dialog" between museums and visitors by enhancing the personalized dimension. Through the semantic database, visitor's preference and interest can be inferred tacitly by observing their behaviour on-site during their interactions with the front-end system. By using the web service, a visitor's personal visit history can also be preserved during the data communication for further analysis or for on-site experience improvement. Below, we outline the framework of this database, and discuss how the semantic metadata can be implemented to establish a personalized tour trajectory.

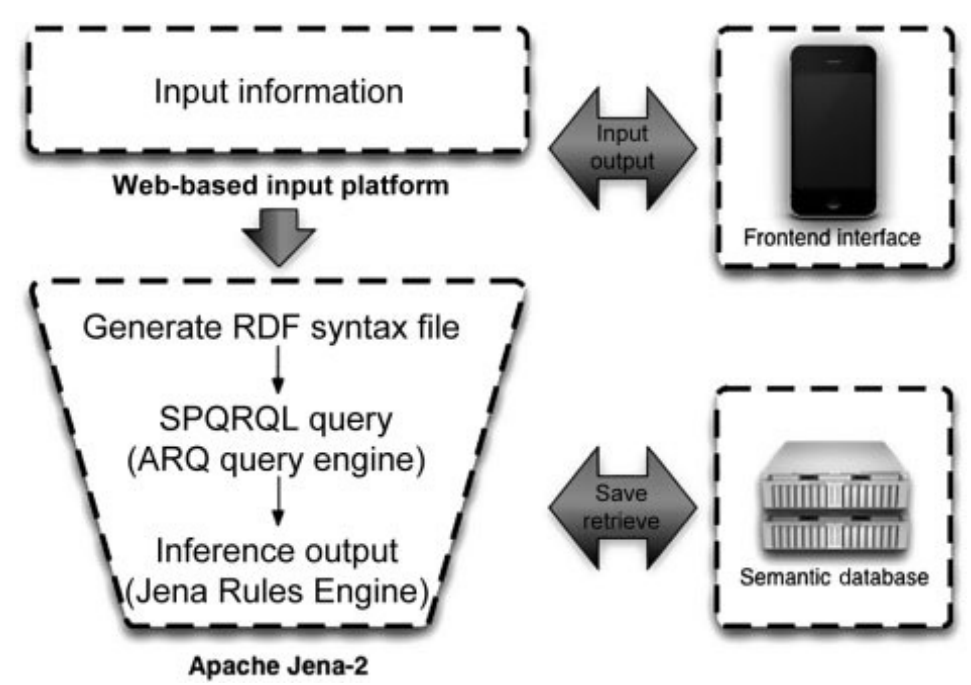

Figure 7. The framework of the back-end semantic database. 
Figure 7 depicts an overview framework of the intermediate web service and back-end semantic database. In this framework, a HTML platform is adopted as an intermediate input/output platform for storing the collections information, visitor's personal visit history, and passing/receiving the data from the semantic database for the handling, processing, inference, and output of result. A short discussion of the components we have adopted to develop the back-end system will be given next.

CIDOC-CRM: Assessing and managing the large collections data are a crucial task for the content management system. In this project, we adopted CIDOC-CRM as the ontology to manage the collections information for database query and inference. As listed in Table 1, several properties have been selected to describe the topics of each collection using this ontology.

Table 1. Mapping between information

\begin{tabular}{l|ll|} 
Descriptions & Properties & Collection Information \\
\hline Author's Name & P52_has_current_owner & Brett WHITELEY \\
Residency & E53_Place & Australia \\
Language & E56_Language & English \\
Influence & P15_was_influenced_by & Francis Bacon \\
Participation & P158 occupied & "Recent Australian Painting" exhibition \\
Site & E27_Site & The Tate Gallery \\
Period I & E4_Period & Cold War \\
Period II & P10_falls_within & Postmodernism and Deconstructivism \\
Year & P4_has_time-span & 1976 \\
Type I & P2_has_type & Painting \\
Type II & E55 Type & still life \\
Technique & P32_used_technique & Oil-painting \\
Classification & P41i_was_classified_by & Social Comment \& Hard Edged Abstraction \\
Technique I & P33_used_specific_technique & Canvas \\
Technique II & P33_used_specific_technique & Oil \\
Location & P7_took_place_at & 5D \\
Measurement/Size & P91_has_unit & 182.0 h x 200.0 w cm \\
Title & P102_has_title & Interior with time past \\
\cline { 2 - 3 } & &
\end{tabular}

Web-based Input platform - The input html platform as shown in Figure 8 is a web-based interface that allows curators and managers to enter information about a collection into the database easily. In this platform, the input data is automatically transformed into RDF/ 
Extensible Markup Language (XML) syntax and mapped to the CIDOC-CRM ontology.

Figure 9 shows an example output of this mapping.

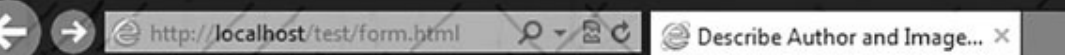

\section{The Forms}

Some information about author and photo.

The Author's Name

(P52_has_current_owner)

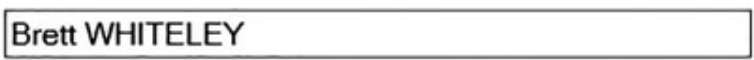

The Year

(P4_has_time-span)

$$
1976
$$

Painting

(P2_has_type)

Classification

(P41i_was_classified_by)

Social Comment \& Hard Edged Abstraction

Canvas

(P33_used_specific_technique)

Technique II

(P33_used_specific_technique)

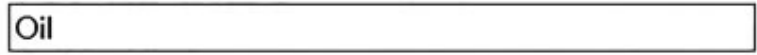

Location

(P7_took_place_at)

$5 \mathrm{D}$

$182.0 \mathrm{~h} \times 200.0 \mathrm{w} \mathrm{cm}$

Measurement/Size

(P91_has_unit)

Title

(P102_has_title)

Interior with time past

\section{GENERATE RDF FILE RESET DATA}

Figure 8. HTML input platform. 


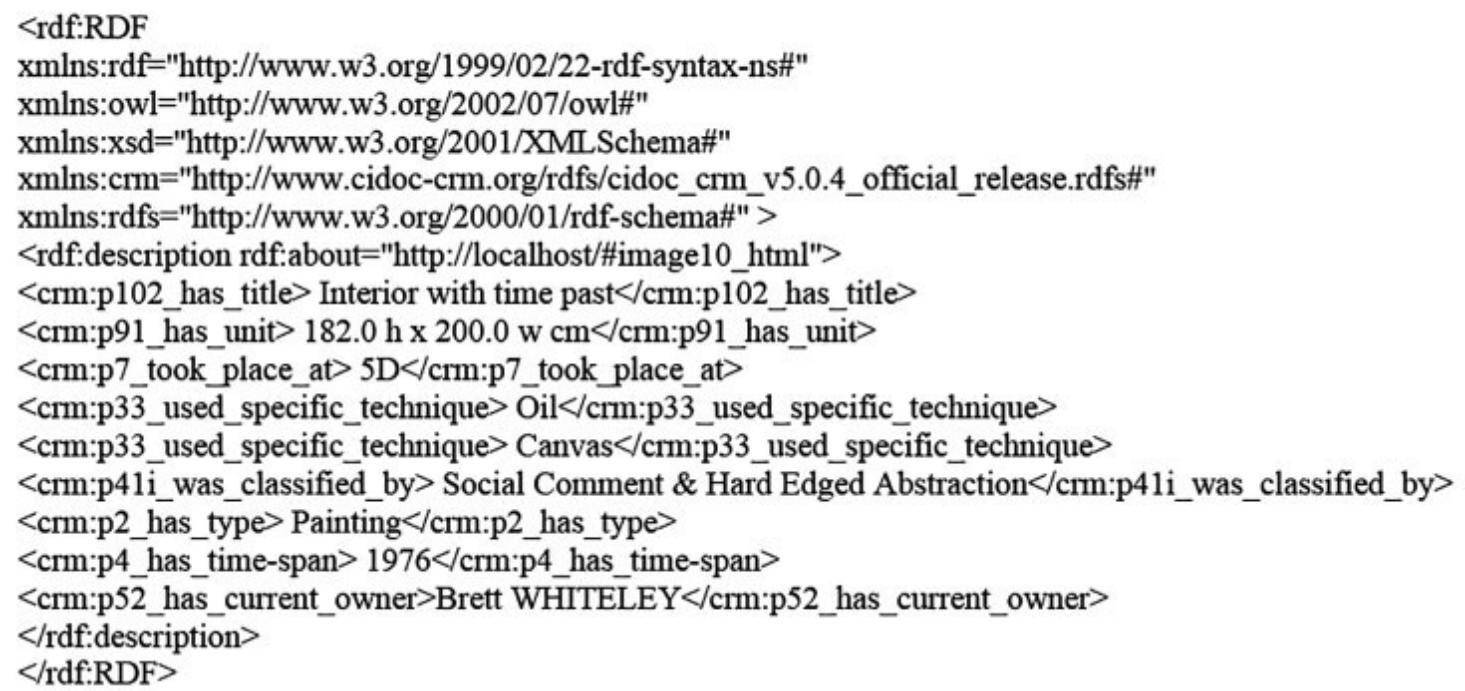

Figure 9. RDF/XML syntax output.

Apache Jena-2 - Apache Jena-2 is an open source Java framework, in which RDF/XML syntax and other types of RDF representation can be created, modified, queried, and inferred by using different Application Programming Interface (APIs) (Min et al. 2005). In Semantic Web, RDF/XML syntax is processed as a set of triples statement, which contains the S-Subject, P-Predicate, and O-Object. When the syntax is created, a series of syntax queries can be made by SPARQL statement via the ARQ query engine. This allows information such as location, title, and size of collection to be used for further reasoning and inference.

\section{System Evaluation}

We evaluated our system by the Cognitive Walkthrough (CW) method with a list of tasks developed based on the cognitive dimensions in this early design stage, in which the usability issues will be addressed. This technique has been widely used in Human-computer interaction (HCI) for understanding the effectiveness of interaction system. According to Wharton and his colleagues (1994), the CW (also called Cognitive Jogthrough) is a task-specific evaluative research method used in the design and deployment periods of interactive systems by 
individual or groups. As Lewis and Rieman (Lewis and Rieman 1993) pointed out, this methodology could benefit developers by identifying the problems of interface design in user-centred design and user personas without the need to actually performing testing using real users. CW was first exploited in the 1990s, focusing on the ease of use for new users to accomplish tasks with a system. The methodology is deployed through five distinct procedures consisted of (i) defining the input to the walkthrough, (ii) defining the tasks to each input, (iii) designing the actions of walking through to each task, (iv) recording critical information from the tasks, and (v) evaluating and discussing the recorded information for further improvement. We will detail each of the procedures by unpacking the tasks that are designed for this system below.

\section{Procedure (i): Defining the input to the Walkthrough}

Clearly circumscribe the input resources is a crucial step of CW method. Wharton has defined four questions for establishing a cognitive scenario at each step, which includes (Wharton et al. 1994):

1. Who will be the users of the system?

2. What task(s) will be analysed?

3. What is the correct sequence for each task and how is it described?

4. How is the interface defined?

The fourth question is initially designed for the paper description of the interface. In this research, we do not need to address this question as the interfaces are already available from our implementation. For the first question, Figure 10 displays the potential users that are targeted by the system within the museum setting. Three groups consisted of general visitors, museums and exhibits curators, and system administrators, are nominated as "users" to sustain the entire framework. However, in consideration of the different knowledge background and technical experiences in each group, we have narrowed down the subjects for evaluation into 
three groups: (1) Visitors who visit museum casually with no technical experiences and art background. (2) Museum and exhibits curators who are equipped with strong aesthetic wisdom and have the ability to manage the collections information. (3) System administrators who have general technical background and are familiar with common programming languages such as JAVA, PHP, and JSP. These subjects are designed to meet the requirements of each group to simulate the various possible situations.

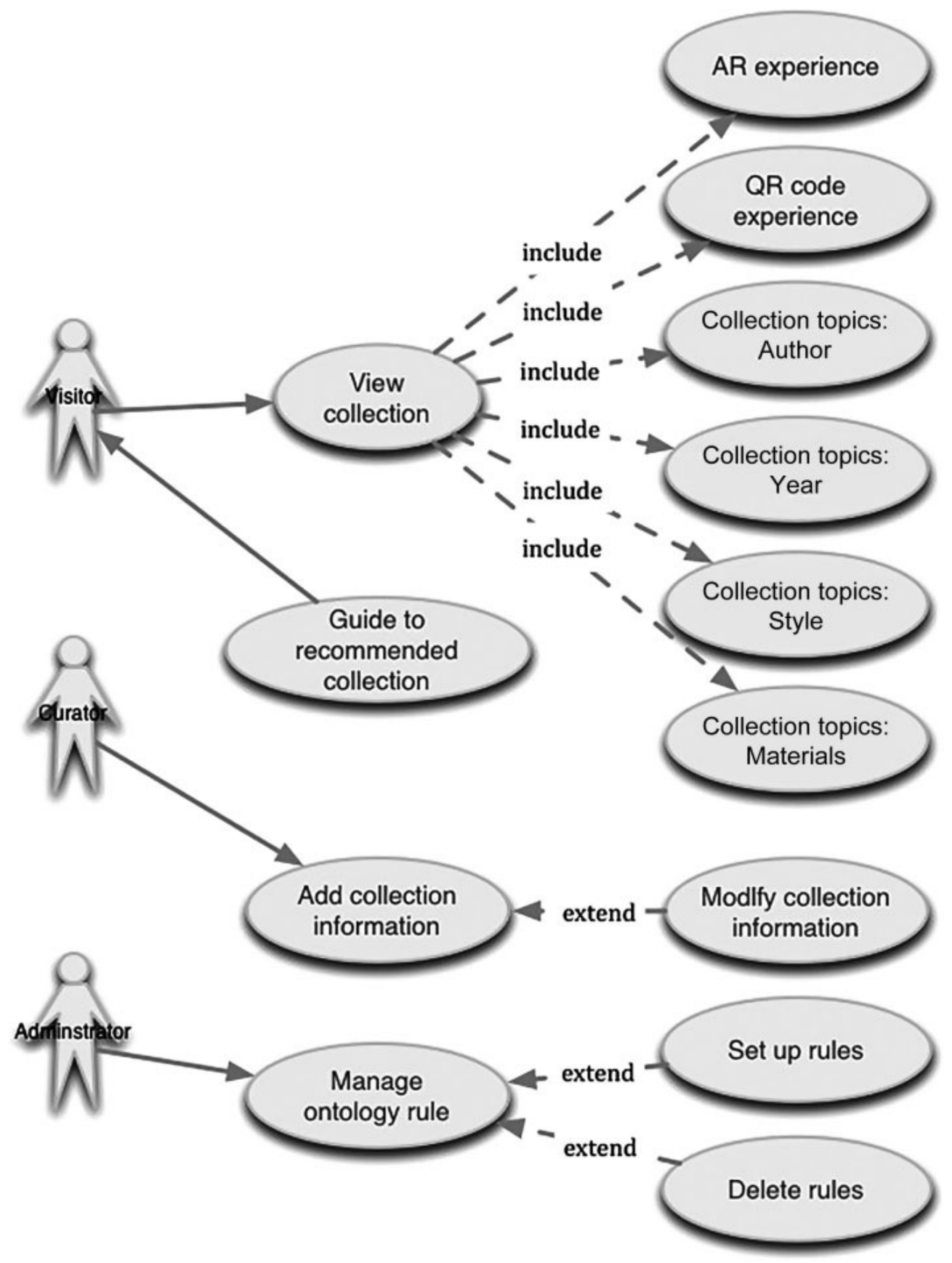

Figure 10. Use case diagram. 


\section{Procedure (ii): Defining the tasks}

The second step is to define the tasks that will be analysed under the cognitive scenario. In order to develop the tasks representatively, we decomposed the tasks into a number of questions based on the cognitive dimensions framework, which was defined by Green and Petre (1996) for evaluating the techniques of interactive devices and designs of programming languages in HCI. The framework was first proposed in 1996 with the composition of 14 dimensions, Abstraction gradient, Closeness of mapping, Consistency, Diffuseness / terseness, Error-proneness, Hard mental operations, Hidden dependencies, Juxtaposability, Premature commitment, Progressive evaluation, Role-expressiveness, Secondary notation and escape from formalism, Viscosity, and Visibility. In this research, we attempted a CW by using these dimensions as the foundation of the tasks for evaluating the effectiveness of user experience. The questions of interest are listed for different groups as below:

For Visitor:

(1) Will the interface be accessed effectively without training?

(2) Will the visitor receive extra information accurately and conveniently during the visit?

(3) Will the information be provided cognitively?

(4) If mistakes are made by operations or decisions, will the visitor be able to go backward?

(5) After the appreciation of current collection, Will the new collection be provided accordingly and proactively?

(6) When observation is progressing, will the visitors understand the feedback they get so they can go to the next step with confidence?

(7) Will the visitor be able to be guided by the system to the next collection?

(8) Will the personal recommendations be made persistently until the end of visit? 
(9) Once the visitors finish the visit, will they receive any feedback regarding the visit?

\section{For Curator:}

(1) Will the collection's information be readily accessible?

(2) Will the curator be able to manage the collection's information according to the exhibits?

(3) Once a visitor finished the visit, will the visitor's behavior be provided for further inspection?

(4) Will the recorded data be valid and analyzable even if the visitor did not finish visiting the entire collections?

For Administrator:

(1) Can the system be maintained without requiring a high-level of technical expertise?

(2) Can the system be created economically using existing technologies?

(3) Has the issue of technological obsolescence in system development been addressed properly?

(4) Can the system be serviced and maintained in an operative condition expeditiously?

(5) After the system is created, will the modules be expandable for further development?

Based on above questions, each group was given a specific task for simulating the cogitative scenario. The task for Visitor was to complete a cycle of visit by using the system for exploring a set of collections, whilst Curator's task was to apply the system to a new exhibit and receive visiting data from each visitor. The task of Administrator would be to update inference rules from the database after the new collections or exhibits were set up by the curator. 


\section{Procedure (iii): The walk through action sequences for each task}

After defining the input subjects and tasks, the actions and sequences of tasks will need to be defined. The action sequences of Visitor denoted as Task1 are:

(a) Select an on-site collection and launch the interface, then click the play button or collection list to enter a collection page.

(b) Freely click the sub-theme pages to navigate through the collection information.

(c) Click the button of augmented reality (AR) and face the camera to the collection for listening or exploring the collection via virtual agent and animation.

(d) Return to the collection page and click "Bring Me To Next Collection" for activating the interactive quiz dialogs.

(e) Click "Yes" on the observation dialogs.

(f) Click "Yes" on the recommendation dialogs.

(g) Click QR code button for searching the current location in association with interactive map navigation to go to the recommended collection.

The actions sequences of Curator denoted as Task2 are:

(a) Launch the input HTML platform and enter or update the new collection information by filling out the blank form.

(b) Click "GENERATE RDF FILE" on the input HTML platform.

(c) Log into the system and download the previous visiting histories from the database.

(d) Analyse the data of past visiting trajectories for optimising future exhibit.

The actions sequences of Administrator denoted as Task3 are:

(a) Launch the Apache Jena via Eclipse.

(b) Choose the existing rules of inference. 
(c) Add or update the rules based on the collections curator has created.

The selected groups go through the whole system for completing the tasks, and the critical information obtained are highlighted in the next section.

\section{Procedure (iv): Recording critical information}

Recording the critical information by walking through the defined tasks can expose the weaknesses of the interactive system and efficiency of the user interface. Lewis and Rieman (1993) recommend four questions for critiquing the tasks:

A. Will the users be trying to produce whatever effect the action has? - It ensures the users can pursue the logical thinking.

B. Will the users be able to notice that the correct action is available? - It evaluates the levels of user-friendliness in the designed interface.

C. Once users find the correct action at the interface, will they know that it is the right one for the effect they are trying to produce? - It ensures the intuitive grasp of each function.

D. After the action is taken, will users understand the feedback they get? - It ensures system can perform a proper feedback after the action is completed.

Keeping these questions in mind, the evaluator will be able to distinguish the success or failure of the tasks, which the participants went through based on the answers to the questions A to D defined by the cognitive dimensions. We evaluated both actions and walkthrough questions in order to obtain critical information from the tasks. Table 2 is the results of execution applying questions A-D to each action. The symbol "Y" (Yes) indicates a well-defined action that can be walked through without extra assistance while an "N" (No) identifies a possible usability problem that may interrupt the potential experience during the interaction. The result $\mathrm{R}$ shows either "P" (Pass) or "F" (Failure). In this table, usability problems on Task1 (c), (g), were identified on questions $\mathrm{A}$ and $\mathrm{B}$, where visitors may skip AR interaction before proceeding to 
the next action, or have difficulty to locate QR function on the screen. Usability problem on

Task2 (b) was identified on questions $\mathrm{C}$ and $\mathrm{D}$ that indicate curators may confuse the name of the generated buttons on the input HTML platform.

Table 2. The execution of tasks

\begin{tabular}{|c|c|c|c|c|c|c|c|c|c|c|c|c|c|c|c|c|}
\hline T1 & (a) & (b) & (c) & (d) & (e) & (f) & (g) & $\mathrm{T2}$ & (a) & (b) & (c) & (d) & T3 & (a) & (b) & (c) \\
\hline A. & $Y$ & $\bar{Y}$ & $\mathrm{~N}$ & $\mathrm{Y}$ & $Y$ & $\mathrm{Y}$ & $\mathrm{N}$ & A. & $Y$ & $\bar{Y}$ & $\mathrm{Y}$ & $\mathrm{Y}$ & A. & $\mathrm{Y}$ & $\mathrm{Y}$ & $Y$ \\
\hline B. & $\mathrm{Y}$ & $\mathrm{Y}$ & $\mathrm{N}$ & $\mathrm{Y}$ & $\mathrm{Y}$ & $\mathrm{Y}$ & $\mathrm{N}$ & B. & $Y$ & $\mathrm{Y}$ & $\mathrm{Y}$ & $\mathrm{Y}$ & B. & $\mathrm{Y}$ & $\mathrm{Y}$ & $\mathrm{Y}$ \\
\hline C. & $\mathrm{Y}$ & $\bar{Y}$ & $\mathrm{Y}$ & $\mathrm{Y}$ & $\mathrm{Y}$ & $\mathrm{Y}$ & $\mathrm{Y}$ & C. & $\bar{Y}$ & $\mathrm{~N}$ & $\mathrm{Y}$ & $\mathrm{Y}$ & C. & $\mathrm{Y}$ & $\mathrm{Y}$ & $\bar{Y}$ \\
\hline D. & $Y$ & $Y$ & $\mathrm{Y}$ & $\mathrm{Y}$ & $\mathrm{Y}$ & $\mathrm{Y}$ & $\bar{Y}$ & D. & $\bar{Y}$ & $\bar{N}$ & $\mathrm{Y}$ & $\bar{Y}$ & D. & $\mathrm{Y}$ & $Y$ & $\bar{Y}$ \\
\hline 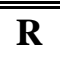 & $\overline{\overline{\mathbf{P}}}$ & $\overline{\bar{P}}$ & $\bar{F}$ & 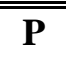 & 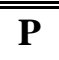 & 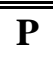 & $\bar{F}$ & $\begin{array}{l}\mathbf{R} \\
\end{array}$ & $\overline{\overline{\mathbf{P}}}$ & $\overline{\bar{F}}$ & 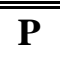 & 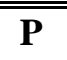 & $\begin{array}{l}\mathbf{R} \\
\end{array}$ & 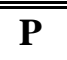 & 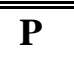 & $\overline{\overline{\mathbf{P}}}$ \\
\hline
\end{tabular}

After the evaluation of each action, the critical information about the walkthrough questions defined in Procedure (ii) will be recorded in the next step. Table 3 listed the results $(\mathrm{Y} / \mathrm{N})$, the affected actions (the bolds are the failed actions), and reasons for the walkthrough questions based on the execution.

Table 3 The critical information of each task.

\begin{tabular}{|c|c|c|c|}
\hline Questions & \multicolumn{2}{|c|}{ Actions } & Reasons \\
\hline & & & Task1: For Visitor \\
\hline \multirow{3}{*}{ (1) } & \multirow{3}{*}{$\mathrm{Y}$} & (a) & \multirow{3}{*}{$\begin{array}{l}\text { The entire system is developed along the UCD principle. On the start page of } \\
\text { the front-end interface, only two buttons are provided for visitors to start } \\
\text { either by selecting the collection of interest from the collection list or starting } \\
\text { from the first collection near the entrance. This design offers the closest way } \\
\text { to simulate the behaviors of both new visitors and experienced visitors when } \\
\text { they visit museum. }\end{array}$} \\
\hline & & (b) & \\
\hline & & & \\
\hline \multirow{3}{*}{ (2) } & \multirow{3}{*}{$\mathrm{N}$} & (b) & \multirow{3}{*}{$\begin{array}{l}\text { The front-end interface supports an extensive amount of collection knowledge } \\
\text { by cataloging the information systematically into different theme pages } \\
\text { However, the use of AR was identified as a usability problem although it } \\
\text { provides an innovative approach for visitors to enhance their art appreciation } \\
\text { and understanding without needing to read the content. }\end{array}$} \\
\hline & & (c) & \\
\hline & & & \\
\hline \multirow{2}{*}{ (3) } & \multirow{2}{*}{$\mathrm{Y}$} & (b) & \multirow{2}{*}{$\begin{array}{l}\text { The collection's information is represented by the different theme pages } \\
\text { according to the features used. As stated by Carretti (2007), this type of } \\
\text { presentation can provide knowledge with cognitive understanding without the } \\
\text { need to memorize information. }\end{array}$} \\
\hline & & & \\
\hline
\end{tabular}




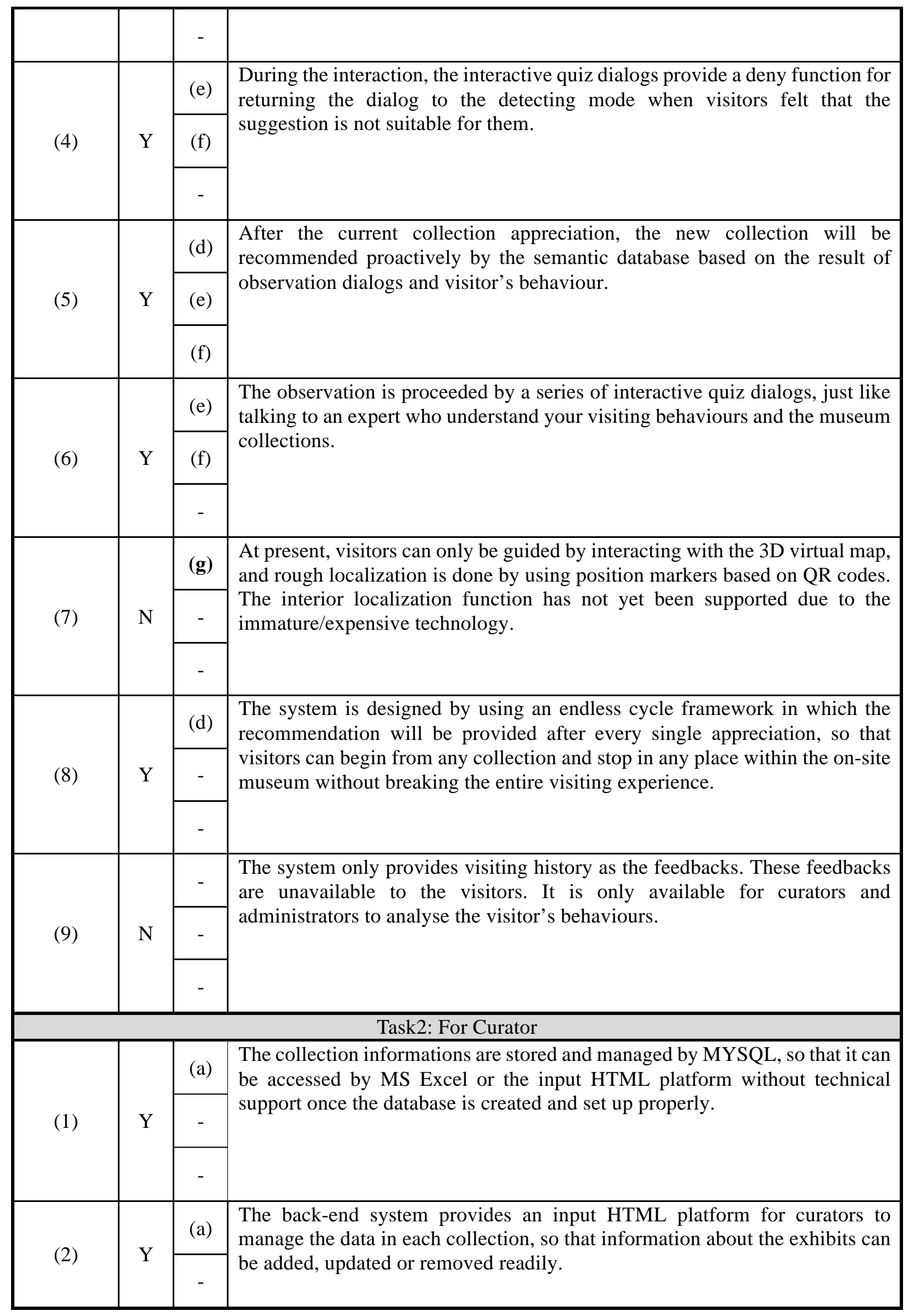




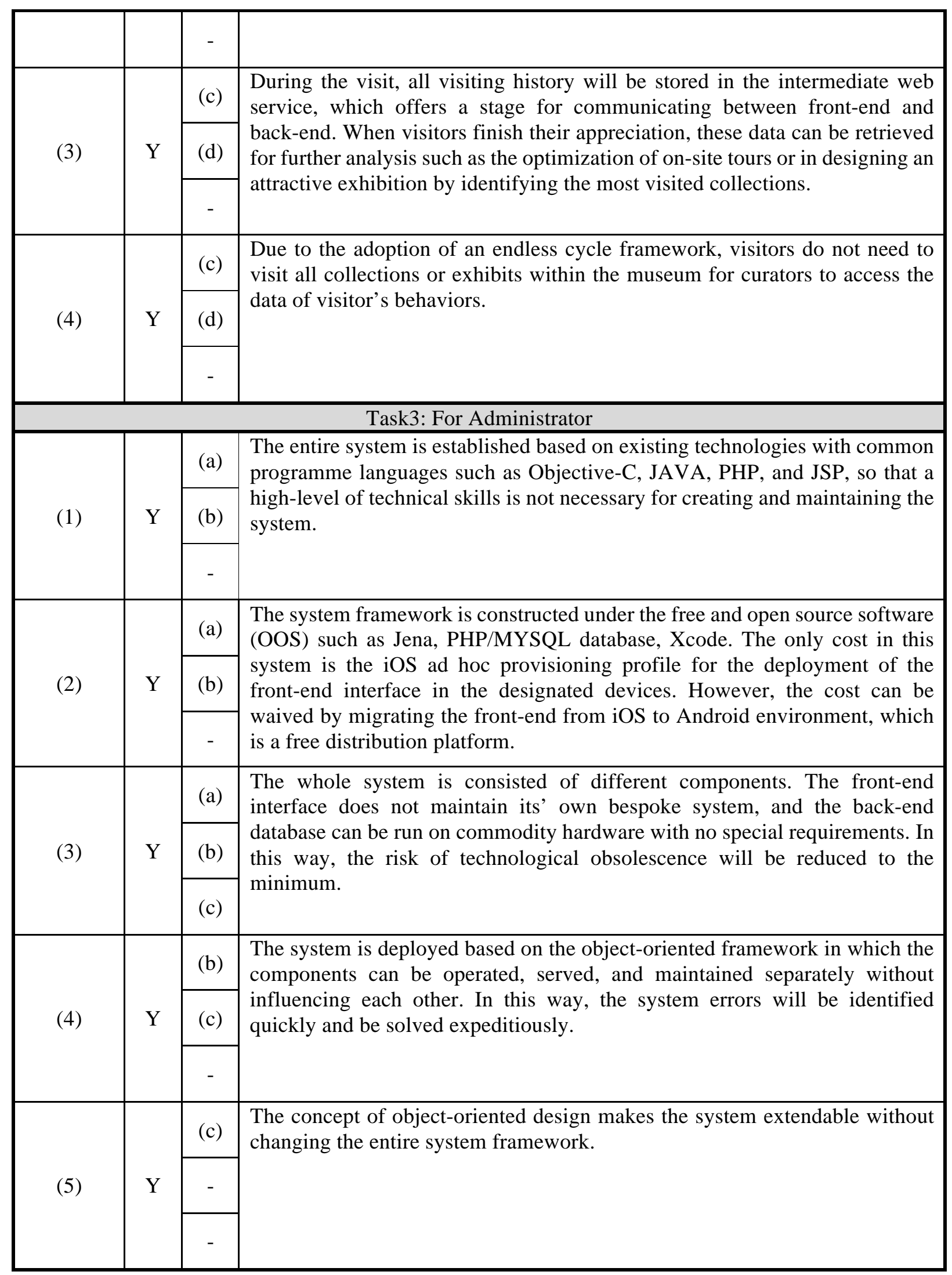




\section{Procedure (v): Result discussion}

As can be seen from the first step of actions evaluation results (Table 2), most of the actions have achieved the expectation of the initial objective except actions "c", "g" in Task1 and "b" in Task2. The issues in Task1 can be addressed by introducing a visual "help" sheet that tells visitor to interact with $\mathrm{AR}$ before proceed to the next step, and starting the QR code function automatically after action "f", while issues in Task 2 can be resolved by changing the nametag on the button.

For questions evaluation in step two (Table 3), although three walkthrough questions in Task1 appear to have issue with respect to the usability problem, the first walkthrough question (Task1, Q2) will be redefined once action "c" has been improved. With the continuous growth of technology, we believe that the second walkthrough question (Task1, Q7) could also be resolved in the near future. For the third walkthrough question (Task1, Q9), even though the visiting history could assist curators to identify visitor's behaviours, the presentation of these information to the visitor are less useful for the visitors themselves.

\section{Conclusion and future development}

Although digital technology has been applied much more extensively in cultural institutions such as museums and art galleries in recent years, very little work has been done on exploring the aspects of on-site interaction and visit personalization in a systematic way. In this article, we describe and evaluate a system to enhance the aspects of interaction and personalization in physical museums and art galleries. Our system enables a personalized museum tour adapting to the interest of different visitor using several enabling technologies through an interactive and dynamic user interface.

In this work, several interesting observations are reflected from the results of action and 
question evaluations in CW. First, the problems identified in action sequence evaluation did not fully impact user experience. Although some actions suffer the usability issues, users can still complete the expected tasks. It suggested that the physical environment in an on-site visit can somehow mitigate the design gaps in digital presentation. For example, although usability problem was identified in the AR function, the on-site collection's description was able to provide the needed information to mitigate the problem. Similarly, even though the system only provides rough location information about the collections, visitors can always access the recommended targets without any major difficulty by interacting with the physical space and virtual maps.

Second, even with only rough localization done through QR code based positioning, indoor navigation still significantly enhanced the visiting experience. In fact, in a confined and controlled environments such as museums or art galleries, a rough but robust localization is often good enough. Moreover, the action of QR code reading in itself added an element of engagement with the physical environment, which is a desirable objective.

Third, the screen size may impact the effectiveness of AR presentation. As our prototype is evaluated based on a smartphone with small screen size instead of a tablet, the AR presentation appears to be less important for the visiting experience.

Fourth, for the interface design, the front-end interface appears to be more influential than the back-end in the system. Although the tasks in the second step of questions evaluation for curator (see Question 2 for curator in Table 3) was able to be completed successfully, a usability issue was perceived for the curator management experience in the first step of action test (Task2, (b)). Finally, although the use of observation dialog could potentially distract the user during art appreciation, it is still perceived by users to be a better way to detect collections of interest over manual collection bookmarking or passive preference selection. Nevertheless, 
poorly designed and overuse of quiz dialog could negate this positive user experience.

Currently, no feedback was provided to the visitors after their visit. With the aim to enhance the after visit experience, in future we plan to provide a personalized tour feedback to the visitors. Besides giving a summary and highlight of their visit, the feedback could also suggest a trajectory for their next visit, thereby encouraging repeated visits to the site. The best way to provide this feedback will be a topic for future investigation.

\section{References}

Andujar, C., A. Chica, and P. Brunet. 2012. “User-Interface Design for the Ripoll Monastery Exhibition at the National Art Museum of Catalonia." Computers \& Graphics 36 (1). Elsevier: 28-37. doi:10.1016/j.cag.2011.10.005.

Angelopoulou, Anastassia, Daphne Economou, Vassiliki Bouki, Alexandra Psarrou, Li Jin, Chris Pritchard, and Frantzeska Kolyda. 2011. “Mobile Augmented Reality for Cultural Heritage." In Mobile Wireless Middleware, Operating Systems, and Applications, edited by Nalini Venkatasubramanian, Vladimir Getov, and Stephan Steglich, 15-22. London, UK: Springer Berlin Heidelberg. doi:10.1007/978-3-642-30607-5_2.

Beer, Valorie. 1987. “Great Expectations: Do Museums Know What Visitors Are Doing?” Curator: The Museum Journal 30 (3). Wiley Online Library: 206-15. doi:10.1111/j.2151-6952.1987.tb00664.x.

Bimber, Oliver, L. Miguel Encarnação, and Dieter Schmalstieg. 2003. The Virtual Showcase as a New Platform for Augmented Reality Digital Storytelling. Proceedings of the Workshop on Virtual Environments 2003 - EGVE '03. New York, New York, USA: ACM Press. doi:10.1145/769953.769964. 
Bowen, J, and S Filippini-Fantoni. 2004. "Personalization and the Web from a Museum Perspective." In Museums and the Web, edited by D. Bearman and J. Trant. Washington DC / Arlington VA, USA: Archives \& Museum Informatics.

BRUSTEIN, JOSHUA. 2011. "An Extra Set of Eyes at the Museum." The New York Times. http://www.nytimes.com/2011/11/20/nyregion/technology-goes-to-the-museum-appcity.html?_r=2.

Carretti, Barbara, Erika Borella, and Rossana De Beni. 2007. “Does Strategic Memory Training Improve the Working Memory Performance of Younger and Older Adults?" Experimental Psychology (formerly "Zeitschrift Für Experimentelle Psychologie") 54 (4): 311-20. doi:10.1027/1618-3169.54.4.311.

Ceipidor, U Biader, C M Medaglia, V Volpi, A Moroni, S Sposato, M Carboni, and A Caridi. 2013. “NFC Technology Applied to Touristic-Cultural Field: A Case Study on an Italian Museum." In 2013 5th International Workshop on Near Field Communication (NFC), 16. IEEE. doi:10.1109/NFC.2013.6482445.

Courage, Catherine, and Kathy Baxter. 2005. Understanding Your Users: A Practical Guide to User Requirements Methods, Tools, and Techniques. Edited by Kathy Baxter. San Francisco, CA: Gulf Professional Publishing.

Economou, Maria, and Elpiniki Meintani. 2011. “Promising Beginnings? Evaluating Museum Mobile Phone Apps." Rethinking Technology in Museums Conference Proceedings.

Falk, John Howard, and Lynn Diane Dierking. 1992. The Museum Experience. Howells House. 
Green, TRG, and M Petre. 1996. "Usability Analysis of Visual Programming Environments: A ‘Cognitive Dimensions' Framework.” Journal of Visual Languages \& Computing 7 (2): 131-74. doi:10.1006/jvlc.1996.0009.

Hoare, Cathal, and Humphrey Sorensen. 2012. “On Automatically Geotagging Archived Images." In Libraries in the Digital Age (LIDA) Proceedings.

Huynh, David, Stefano Mazzocchi, and David Karger. 2007. “Piggy Bank: Experience the Semantic Web inside Your Web Browser." Web Semantics: Science, Services and Agents on the World Wide Web 5 (1): 16-27. doi:10.1016/j.websem.2006.12.002.

LeVitus, B. 2010. “Musée Du Louvre.” In Incredible iPhone Apps For Dummies, 36-37. Canade: Wiley Publishing, Inc.

Lewis, Clayton, and J Rieman. 1993. Task-Centered User Interface Design: A Practical Introduction. Task-Centered User Interface Design: A Practical Introduction. University of Colorado, Boulder, Department of Computer Science.

Lynch, Elizabeth. 2011. "Commissions." Sculpture, March.

Min, Wang, Ding Jianping, Xiang Yang, and Xun Henxing. 2005. "The Research on the Jena-Based Web Page Ontology Extracting and Processing." In 2005 First International Conference on Semantics, Knowledge and Grid, 105-105. Beijing: IEEE. doi:10.1109/SKG.2005.128.

Oppermann, Reinhard. 2002. "User-Interface Design." In Handbook on Information Technologies for Education and Training, edited by Heimo H. Adelsberger, Betty Collis, 
and Jan M. Pawlowski, 233-48. Springer Berlin Heidelberg. doi:10.1007/978-3-662-07682-8_15.

Proctor, N, and J Burton. 2004. "Tate Modern Multimedia Tour Pilots 2002-2003." MLEARN 2003: Learning with Mobile Devices, 1-4.

Schreiber, Guus. 2010. "Web Science: The Digital-Heritage Case." SOFSEM 2010: Theory and Practice of Computer Science 5901. Springer: 108-11. doi:10.1007/978-3-642-11266-9_9.

Tallon, Loïc. 2013. "Mobile Strategy in 2013: An Analysis of the Annual Museums \& Mobile Survey."

Tallon, Loïc. 2008. Digital Technologies and the Museum Experience: Handheld Guides and Other Media. Rowman Altamira.

Wang, Ching-Sheng, Ding-Jung Chiang, and Yi-Yun Ho. 2012. "3D Augmented Reality Mobile Navigation System Supporting Indoor Positioning Function." In 2012 IEEE International Conference on Computational Intelligence and Cybernetics (CyberneticsCom), 64-68. IEEE. doi:10.1109/CyberneticsCom.2012.6381618.

Weiner, Sara. 2011. "RTCRM Perspectives.” Vol. 2. www.rtcrm.com/blog/perspectives2011.

Wharton, Cathleen, John Rieman, Clayton Lewis, and Peter Polson. 1994. "The Cognitive Walkthrough Method: A Practitioner's Guide." Usability Inspection. doi:10.1108/09685220910944731. 
Wojciechowski, Rafal, Krzysztof Walczak, Martin White, and Wojciech Cellary. 2004.

"Building Virtual and Augmented Reality Museum Exhibitions." In Proceedings of the Ninth International Conference on 3D Web Technology - Web3D '04, 135. New York, New York, USA: ACM Press. doi:10.1145/985040.985060. 